\title{
Identification of lactic acid bacteria in the rumen and feces of dairy cows fed total mixed ration silage to assess the survival of silage bacteria in the gut
}

\author{
H. Han, ${ }^{*}$ Y. Ogata, ${ }^{*}$ Y. Yamamoto,† S. Nagao, $\ddagger$ and N. Nishino*1 \\ *Department of Animal Science, Okayama University, Okayama 700-8530, Japan \\ †Mie Prefecture Livestock Research Institute, Mie 515-2324, Japan \\ ‡Okayama Prefecture Livestock Research Institute, Okayama 709-3494, Japan
}

\section{ABSTRACT}

The survival of silage lactic acid bacteria (LAB) in the gut of dairy cows was evaluated by examining the LAB communities of silage and gut contents. Samples were collected at 2 different research institutes (Mie and Okayama) that offered total mixed ration (TMR) silage throughout the year. Silage and feces were sampled in August, October, and November at the Mie institute, whereas silage, rumen fluid, and feces were sampled in June and August at the Okayama institute. Denaturing gradient gel electrophoresis using Lactobacillusspecific primers was performed to detect LAB species in the samples. The selected bands were purified for species identification and the band patterns were used for principal component analysis. Lactic acid was the predominant fermentation product in all the TMR silages analyzed, and the lactic acid level tended to be constant regardless of the sampling time and region. A total of 14 LAB species were detected in the TMR silage samples, of which 5 (Lactobacillus acetotolerans, Lactobacillus pontis, Lactobacillus casei, Lactobacillus suebicus, and Lactobacillus plantarum) were detected in the dairy cow feces. Most of the denaturing gradient gel electrophoresis bands for the feces samples were also detected in the rumen fluid, suggesting that any elimination of silage LAB occurred in the rumen and not in the postruminal gut segments. The principal component analysis indicated that the LAB communities in the silage, rumen fluid, and feces were separately grouped; hence, the survival of silage LAB in the cow rumen and lower gut was deemed difficult. It was concluded that, although the gut LAB community is robust and not easily affected by the silage conditions, several LAB species can inhabit both silage and feces, which suggests the potential of using silage as a vehicle for conveying probiotics.

Key words: dairy cow, gut, lactic acid bacteria, silage

Received January 21, 2014

Accepted May 10, 2014.

${ }^{1}$ Corresponding author: j1oufeed@cc.okayama-u.ac.jp

\section{INTRODUCTION}

Lactic acid bacteria (LAB) are of primary importance in the process of moist forage crop preservation. Energy losses and protein degradation can be reduced if LAB, particularly homo-fermentative species, predominate the fermentation process (McDonald et al., 1991). Inoculants, such as Lactobacillus plantarum, Lactobacillus acidophilus, Enterococcus faecium, and others, are often used to secure desirable lactic acid fermentation (Weinberg and Muck, 1996). After long-term inoculant use, the primary purposes, such as reducing nutrient loss and preventing intensive proteolysis, have almost been achieved. Accordingly, the focus of attention has now shifted to additional functions, such as promoting animal health and disease prevention. As LAB constitutes a part of the ruminant gut bacteria, silage can be considered a vehicle to propagate and deliver probiotic LAB species. Although the original concept regarding probiotics is based on benefits taking place postruminally, certain probiotics may even confer advantages in the rumen, such as improved digestibility and an inhibition of acidosis (McAllister et al., 2011). Animal performance (intake, weight gain, and milk yields) could thus be enhanced with LAB-inoculated silage even when no fermentation improvement is seen; however, the ability for improvement is dependent on the strains used (Weinberg and Muck, 1996).

To ensure that a probiotic-based inoculation is beneficial, the survival of LAB in the ruminant gut needs to be verified. Weinberg et al. (2004) examined the changes in the populations of silage LAB (L. plantarum, E. faecium, and Pediococcus pentosaceus) during an in vitro rumen incubation and concluded that acceptable numbers of LAB could survive, particularly when sugar substrates were used for fortification (Weinberg et al., 2004). Similarly, Rodriguez-Palacios et al. (2009) isolated L. plantarum from the cecum and both $P$. pentosaceus and Pediococcus acidilactici from bovine fecal matter, suggesting that LAB species used as inoculants may survive in both the rumen as well as the intestine. However, as Rodriguez-Palacios et al. (2009) 
acknowledged, the isolation of L. plantarum from the bovine gut is rare. The LAB species usually detected in the gut are Streptococcus bovis, Lactobacillus vitulinus, Lactobacillus ruminis, Lactobacillus johnsonii, and Lactoabacillus murinus (Krause et al., 2003; Hernandez et al., 2008; Nader-Macías et al., 2008). This raises a debate on how to select LAB species for developing probiotic supplements.

To determine the survival of silage LAB in the bovine gut, we performed a practical survey to monitor the LAB community in bunker-made whole crop corn silage and in the feces of dairy cows receiving the silage $[\mathrm{H}$. Han, C. Wang (Okayama University, Okayama, Japan), Z. Yu (China Agricultural University, Beijing, China), Q. Xu (China Agricultural University), and N. Nishino, unpublished data]. Three (Lactobacillus acetotolerans, Lactobacillus pontis, and Lactobacillus casei) out of 8 silage LAB species were detected in feces, suggesting that although it may be tough for silage LAB to survive the digestive process in the gut, several LAB species may have the potential to act as probiotics when supplemented with silage to dairy cows.

In the previous survey, farmers used corn silage at a proportion of 20 to $40 \%$ in the dairy cow diet. Silage LAB were diluted by mixing with other feeds, whereas concentrated feeds are known to acidify the rumen content and thereby increase the competition between LAB and other gut bacteria. Meanwhile, survival of the silage LAB can be scrutinized in greater detail if the ruminal bacterial community is examined together with that of the feces. If silage LAB are not detected in the feces, it remains undetermined whether their elimination takes place ruminally or postruminally. In Japan, production and feeding of TMR silage, a silage that stores the entire mass of TMR mixture, has been practiced. Based on the feeding regimen of TMR silage, gut content samples can be collected from dairy cows that were exclusively fed silage. In our study, the fate of silage $\mathrm{LAB}$ was evaluated by examining the $\mathrm{LAB}$ community in TMR silage, rumen fluid, and fecal material of dairy cows to see whether the silage LAB are removed ruminally or postruminally.

\section{MATERIALS AND METHODS}

\section{TMR Silage, Rumen Fluid, and Fecal Sampling from Dairy Cows}

We collected samples of TMR silages produced at the Mie Prefecture Livestock Research Institute (4 bales each on August 23, October 29, and November 2, 2010) and a feed company based in the Okayama prefecture (2 bales each on June 18 and August 20, 2012). The composition of the Mie TMR silage varied depending on production time (Table 1). Three crop (corn, sorghum, and wilted Italian ryegrass) silages were used in the August product, whereas only single crop (wheat) and crop-free silages were used in the October and November products, respectively. For the November product, timothy hay was used instead of crop silage. The CP and total digestible nutrient levels were set at $\sim 15$ and $\sim 72 \%$ DM by mixing the silage with concentrates and wet by-products. The TMR mixture was then wrapped with 6 layers of plastic film (Shito et al., 2006). For the Okayama TMR products, corn and rice silages were used as ingredients and the recipe was unaltered between the June and August preparations. The nutrient composition was similar to that of the Mie products; however, the Okayama prefecture-derived TMR mixture was stored after vacuum-sealing in a thick (0.1 $\mathrm{mm}$ ) plastic bag. The mixtures were stored outside for 1 to 2 mo in both regions. Several grab samples were mixed to create a composite sample of $\sim 0.5 \mathrm{~kg}$.

At the Mie Livestock Research Institute, TMR silage was offered ad libitum to dairy cows throughout the year and rectal samples were taken at around $1000 \mathrm{~h}$. As fecal samples collected from 3 cows on 3 consecutive days showed no apparent day-to-day variations in the denaturing gradient gel electrophoresis (DGGE) patterns, fecal samples taken on the first day were used for community analysis as representative samples. About $1 \mathrm{~g}$ of feces was aliquoted in an Eppendorf tube and shipped frozen to Okayama University. Because some of the cows were dried off between August and November, occasional differences were observed among the dairy cows depending on the collection time.

For the Okayama products, silages were transported from the feed company and stored at the Okayama Prefecture Livestock Research Institute until use. Similar to the Mie Institute, TMR silage was also offered ad libitum to dairy cows throughout the year. We collected the rumen content and rectal samples at around $1300 \mathrm{~h}$ from 5 dairy cows. The rumen content was obtained using a flexible stomach tube and then strained through 4 layers of surgical gauze. About $1 \mathrm{~g}$ of the rumen fluid and fecal samples were placed in Eppendorf tubes, shipped on ice, and were stored frozen until analysis at Okayama University. Because the 2 sampling times were close, the rumen fluid and feces were taken from the same dairy cows.

\section{Chemical and Bacterial Community Analyses}

The silage DM content was determined by oven drying at $60^{\circ} \mathrm{C}$ for $48 \mathrm{~h}$. The fermented product content in the silage was determined following water extraction using an ion-exclusion polymeric HPLC method with refractive index detection (Han et al., 2012). 
Table 1. Composition and calculated nutrient content of TMR silage produced in various seasons in the Mie and Okayama regions

\begin{tabular}{|c|c|c|c|c|}
\hline \multirow[b]{2}{*}{ Item } & \multicolumn{3}{|c|}{ Mie } & \multirow{2}{*}{$\begin{array}{c}\text { Okayama } \\
\begin{array}{c}\text { June and } \\
\text { August }\end{array}\end{array}$} \\
\hline & August & October & November & \\
\hline \multicolumn{5}{|l|}{ Ingredient (\% DM basis) } \\
\hline Whole crop corn silage & 7.06 & - & - & 16.5 \\
\hline Sorghum silage & 7.88 & - & - & - \\
\hline Wilted Italian ryegrass silage & 23.4 & — & - & - \\
\hline Whole crop wheat silage & - & 31.5 & - & - \\
\hline Whole crop rice silage & - & - & - & 6.54 \\
\hline Brewers grain silage & - & 6.16 & 6.29 & - \\
\hline Alfalfa hay & - & 5.29 & 5.78 & 14.6 \\
\hline Sudan grass hay & - & - & - & 18.3 \\
\hline Timothy hay & - & - & 31.6 & - \\
\hline Beet pulp & 8.04 & 6.49 & 3.40 & 15.2 \\
\hline Rolled corn & 18.8 & 17.7 & 19.9 & 10.1 \\
\hline Rolled barley & 4.51 & 7.55 & 9.92 & - \\
\hline Wheat bran & 4.44 & 7.63 & 6.67 & - \\
\hline Soybean (wet heat) & 4.80 & 4.11 & 1.20 & 2.75 \\
\hline Soybean meal flakes & 13.5 & 6.44 & 9.02 & 2.58 \\
\hline Corn gluten feed & 4.51 & 5.15 & 4.50 & 2.62 \\
\hline Cottonseed & - & - & - & 2.68 \\
\hline Corn steep liquor & - & - & - & 1.39 \\
\hline Molasses & - & - & - & 4.28 \\
\hline Minerals & 2.78 & 1.81 & 1.58 & 2.54 \\
\hline Mycofix $^{2}$ & 0.25 & 0.22 & 0.19 & - \\
\hline \multicolumn{5}{|l|}{ Calculated nutrients (\% of DM) } \\
\hline Total digestible nutrients & 72.8 & 72.2 & 72.2 & 70.4 \\
\hline $\mathrm{CP}$ & 16.1 & 15.3 & 15.4 & 15.3 \\
\hline
\end{tabular}

${ }^{1}$ Water was added at the time of mixing to rectify the moisture content of the Mie November product and the Okayama June and August products.

${ }^{2}$ Japan Nutrition Co. Ltd. (Tokyo, Japan).

The DGGE was performed as previously described (Han et al., 2012). Extraction and purification of silage bacterial DNA was performed using a commercial kit (DNeasy Tissue kit; Qiagen, Germantown, MD). The rumen fluid and fecal samples were purified using the mini DNeasy Stool kit (Qiagen). Nested PCR was employed on the DNA obtained to determine the Lactobacillus-specific community. The protocol involved an initial PCR with Lab 159f (5'-GGAAACAG (A/G) TGCTAATACCG-3') and Lab 677r (5'-CACCGCTACACATGGAG-3') primers, followed by a second PCR with GC-containing universal primers: GC357f (5'-CGCCCGCCGCGCGCGGCGGGCGGGGCGGG GGCACGGGGGGCCTACGGGAGGCAGCAG-3') and 517r (5'-ATTACCGCGGCTGCTGG-3').

The first round of PCR was performed with an initial denaturation at $94^{\circ} \mathrm{C}$ for $2 \mathrm{~min}$, followed by 35 amplification cycles (denaturation at $94^{\circ} \mathrm{C}$ for $30 \mathrm{~s}$, annealing at $61^{\circ} \mathrm{C}$ for $1 \mathrm{~min}$, and extension at $68^{\circ} \mathrm{C}$ for $1 \mathrm{~min}$ ), and a final extension at $68^{\circ} \mathrm{C}$ for $7 \mathrm{~min}$. In the second round, an initial denaturation was performed at $95^{\circ} \mathrm{C}$ for $10 \mathrm{~min}$, followed by 30 amplification cycles [denaturation at $93^{\circ} \mathrm{C}$ for $30 \mathrm{~s}$; annealing at $65^{\circ} \mathrm{C}$ (first 10 cycles), $60^{\circ} \mathrm{C}$ (second 10 cycles), or $55^{\circ} \mathrm{C}$ (last 10 cycles) for $30 \mathrm{~s}$, and an extension step at $72^{\circ} \mathrm{C}$ for $\left.1 \mathrm{~min}\right]$, and a final extension at $72^{\circ} \mathrm{C}$ for $5 \mathrm{~min}$.

The GC-clamped PCR products were separated based on their sequences using the DCode Universal Mutation Detection System (Bio-Rad Ltd., Tokyo, Japan). Samples were applied directly onto $10 \%$ (wt/ vol) polyacrylamide gels, which were prepared with a denaturing gradient (20-50\% for the Mie samples and $25-50 \%$ for the Okayama samples) of urea and formamide [7 $M$ urea and $40 \%$ (vol/vol) formamide as the $100 \%$ denaturant]. Electrophoresis was conducted at a constant voltage of $150 \mathrm{~V}$ for $12 \mathrm{~h}$ at $60^{\circ} \mathrm{C}$. After electrophoresis, gels were stained using SYBR Green (CambrexBio Science Inc., Rockland, ME) and photographed under UV illumination.

\section{Cloning and Sequencing of the DGGE Bands}

Selected bands were excised from the DGGE gels and placed in $10 \mu \mathrm{L}$ of sterilized water at $4^{\circ} \mathrm{C}$ overnight to allow for DNA diffusion. Extracted DNA was then PCR-amplified using the $357 f$ (without GC-clamp) and 517r primers, purified PCR products were cloned into the pTAC-1 vector, and the resulting plasmids were 
transformed into competent Escherichia coli DH5 $\alpha$ cells (DynaExpress TA cloning kit, BioDynamics Laboratory Inc., Tokyo, Japan). The sequencing reaction was conducted using a BigDye Terminator v3.1 Cycle Sequencing Kit (Applied Biosystems Inc., Foster City, $\mathrm{CA}$ ), and the DNA sequences were analyzed using an ABI PRISM 3100 sequencer (Applied Biosystems Inc.).

\section{Species Identification and Nucleotide Sequence Accession Numbers}

Bacterial species were identified by BLAST searches using the GenBank database (http://blast.ncbi.nlm. nih.gov/Blast.cgi), and the closest relatives based on partial 16S rRNA gene sequences were determined. The test and BLAST-identified sequences were considered similar when more than $99 \%$ of the test sequence was identical to that in the BLAST database. The rRNA gene sequences determined in this study have been deposited in the DNA Data Bank of Japan (accession numbers AB853939-AB853995).

To compare the differences among the LAB communities in silage, rumen fluid, and feces, and between 2 sampling times, the DGGE band profiles were analyzed using an image analysis system (Image J; http://imagej.nih.gov/ij/). The migration patterns of the DNA bands were converted into a series of binary numbers, and principal component analysis using 25 (Mie samples) and 26 (Okayama samples) bands was performed using the JMP software (ver. 7; SAS Institute, Tokyo, Japan).

\section{RESULTS}

The lactic acid content (5.49-5.95\% of DM) was similar in all of the silage samples examined (Table 2 ). The acetic acid content was lower in the Mie November product compared with the Mie August and
October products and the Okayama products. The lactic-to-acetic acid ratio was thus the highest in the Mie November product. We detected 2,3-butandiol in 2 Mie products, whereas ethanol was detected in all TMR silages regardless of the product origin. Among the silages, the Mie October product showed the highest 2,3-butanediol (1.09\% of DM) and ethanol (3.63\% of DM) levels. Except for the Okayama June product, 1,2-propandiol was detected in all TMR silages, with the highest levels observed in the Mie August product (1.25\% of DM). 1-Propanol was detected in all TMR silages, and no significant differences were seen across product sampling seasons and sampling regions.

The LAB communities between the 4 different bales were similar, whereas distinct seasonal variation was observed in the Mie products (Figure 1). Lactobacillus acetotolerans (bands 1 and 5), Lactobacillus parafarraginis (band 2), P. acidilactici (band 3), Lactobacillus buchneri (band 4), Pediococcus ethanolidurans (band 6), and Lactobacillus acidipiscis (the same position as band 11) were found in the August products. Many bands detected in the October product DGGE analyses were also observed in the August product analyses; however, bands for L. parafarraginis and P. acidilactici were not detected in October product analysis. In contrast, the November product band patterns differed from those of the other Mie products. In addition to P. acidilactici (band 18) and L. buchneri (the same position as band 4), Lactobacillus suebicus (bands 19), Weissella paramesenteroides (bands 20 and 23), Lactobacillus helveticus (band 21), and Lactobacillus casei (band 22) were detected as LAB species exclusive to the November products.

The LAB communities in dairy cow feces appeared stable. Lactobacillus animalis (band 8), Lactobacillus johnsonii (band 13), and Lactobacillus reuteri (band 10), which were not detected in TMR silage, were found regardless of the sampling time. A band for $L$.

Table 2. Dry matter content, $\mathrm{pH}$ value, and fermentation product concentrations of TMR silage produced and fed to dairy cows in the Mie and Okayama regions ${ }^{1}$

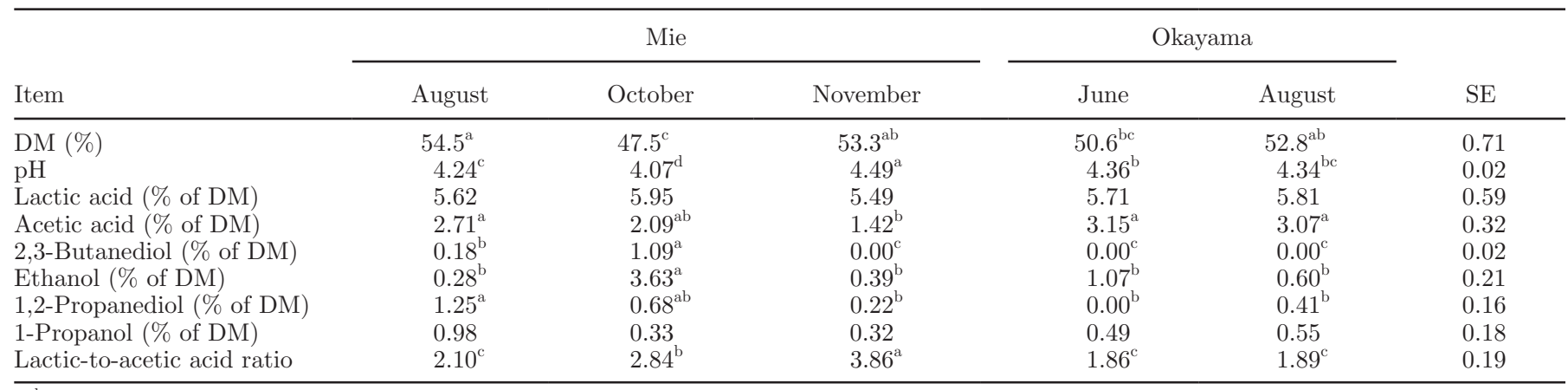

${ }^{\mathrm{a}-\mathrm{d}}$ Means within a same row with different superscript letters differ at $P<0.05$.

${ }^{1}$ Three (Mie) and 2 (Okayama) bags of silage were examined for each product season. 


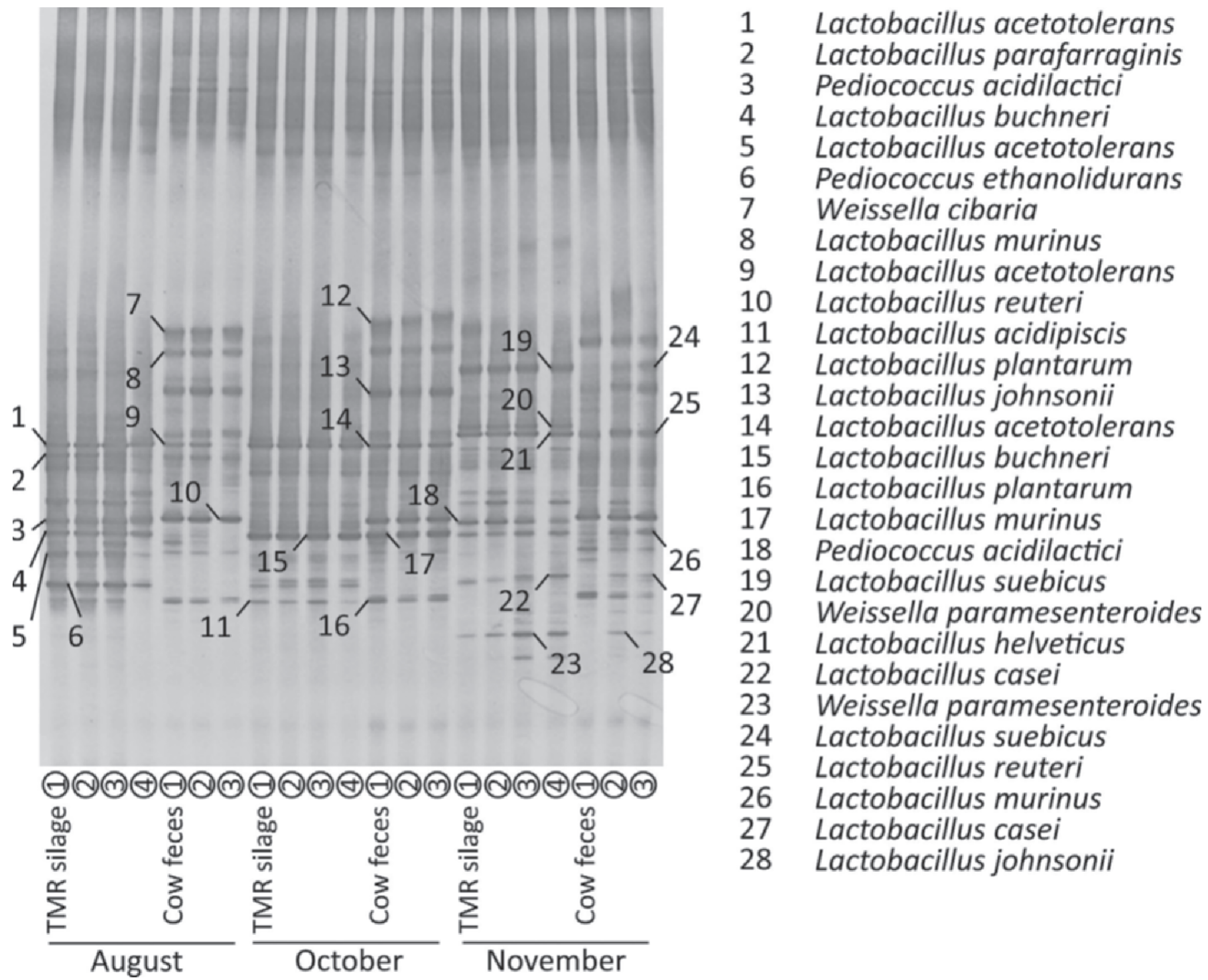

Figure 1. Lactic acid bacteria communities of TMR silage and dairy cow feces collected at 3 different times from the Mie Prefecture Livestock Research Institute (Mie, Japan). The seasons describe when the silage and feces were sampled; silage was produced 1 to 2 mo before the sampling time.

plantarum (band 16) was also seen consistently following analysis of dairy cow feces. Although band 11 in August and October products of TMR silage appeared at the same migrating position as band 16 , the LAB species identified was Lactobacillus acidipiscis (band 11), and no bands corresponding to bands 11 and 16 were present in the November product of TMR silage. The LAB species that appeared to survive in the gut of dairy cows were $L$. acetotolerans (bands 9 and 14), $L$. suebicus (band 24), and L. casei (band 27). Although the position of several bands was close to that of $L$. buchneri, they were identified as L. animalis (bands 17 and 26) in the Mie dairy cow feces.
The principal component analysis demonstrated that although close groups were formed in the August and October products and in the dairy cows fed with those products, the LAB species in silage and feces were grouped separately, indicating that the silage LAB composition was not a critical factor in the formation of the fecal LAB population (Figure 2a). Meanwhile, the LAB communities in the November product and in the dairy cows receiving this product appeared to form groups apart from the others. Taken together, these results suggest that the differences in the silage LAB community could have only marginal influence on the fecal LAB of dairy cows. 
The Okayama product band patterns did not vary based on the season of collection and no interbag variation was observed (Figure 3). Lactobacillus pontis (bands 29, 31, 32, and 33), Lactobacillus amylolyticus (band 30 ), L. reuteri (band 34), L. plantarum (band 35), W. paramesenteroides (band 36), and L. acetotolerans (bands 37 and 53) were found consistently. The LAB communities in the rumen fluid collected from 5 dairy cows at both sampling times showed almost no variation. Bands for L. plantarum (band 42) and L. acetotolerans (band 54) were detected at the same migrating position as those found following TMR silage analysis. However, DNA samples isolated from bands 39 and 41, which were identified as $L$. reuteri and $L$. pontis, respectively, in TMR silage, were identified as uncultured bacterium in the rumen fluid. Furthermore, although the DNA molecules detected in silage and rumen fluid (bands 35 and 42) were identified as L. plantarum, they were designated as an uncultured bacterium following analysis of the corresponding DNA from fecal samples (band 51). Likewise, although the DNA molecules corresponding to L. pontis were found in silage and feces (bands 33 and 48), the DNA seen at the same migrating position in the rumen fluid was found to correspond to an uncultured bacterium (band 41). Meanwhile, gel staining demonstrated that diversity of the gut bacteria (as indicated by the number of DGGE bands) was less for feces than for rumen fluid (i.e., most bands detectable in feces had corresponding bands in the rumen fluid). Lactobacillus acetotolerans was the only LAB species that was consistently identified in TMR silage, rumen fluid, and feces (bands 53, 54, and 57).

The silage LAB communities were closely grouped regardless of the sampling time (Figure $2 \mathrm{~b}$ ). The rumen fluid LAB communities were distributed rather broadly and the June and August samples appeared to form separate groups, indicating sampling time-dependent variations in the rumen LAB among individual dairy cows. The fecal LAB communities appeared to vary even more, depending on the sampling time. The principal component analysis results demonstrate that the LAB community in the rumen fluid or in the feces of dairy cows was not directly related to that in the silage.

\section{(a) Silage vs. feces (Mie samples)}

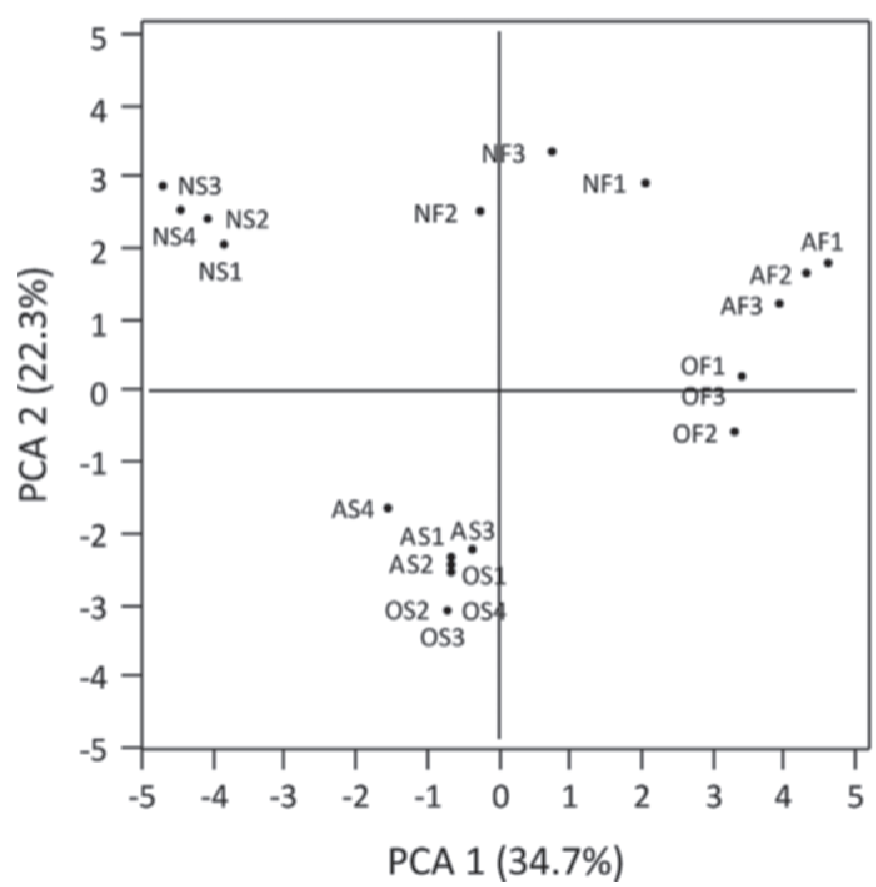

\section{(b) Silage, rumen fluid vs. feces (Okayama samples)}

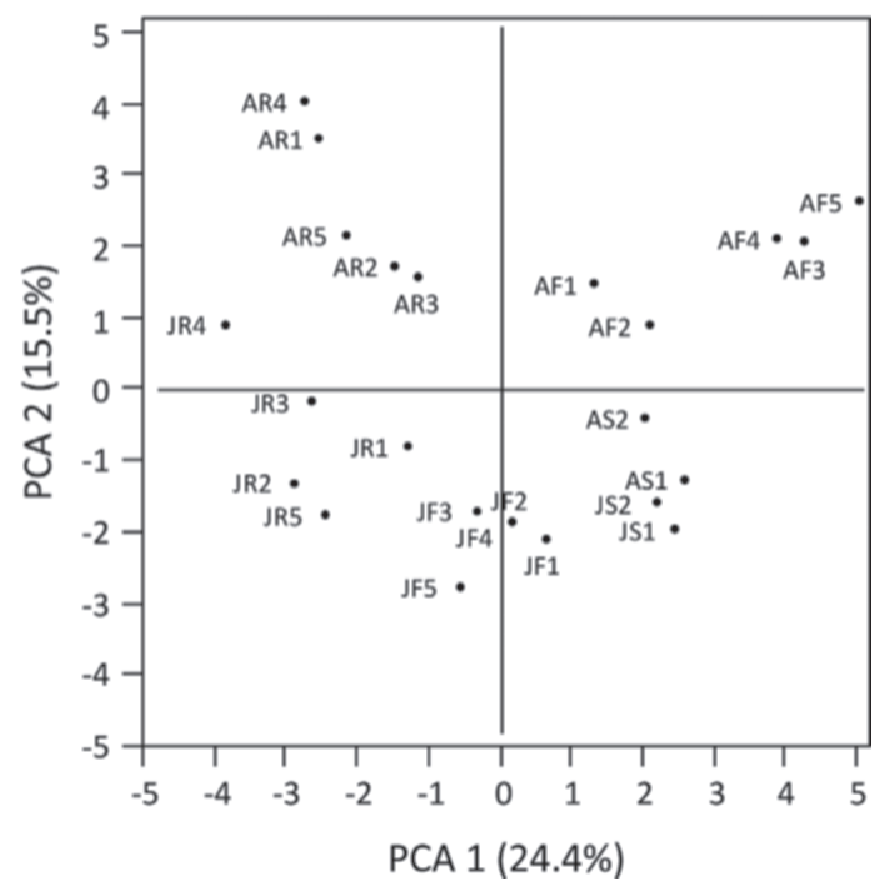

Figure 2. The principal component analysis (PCA) of the lactic acid bacteria community by using denaturing gradient gel electrophoresis (DGGE) profiles of (a) Mie samples of TMR silage and feces of the silage-fed dairy cows and (b) Okayama samples of TMR silage and rumen fluid and feces of the silage-fed dairy cows. For the Mie samples, the first letter stands for August (A), October (O), and November (N); the second letter for silage (S) and feces (F); and the number for a silage bag and dairy cow. For the Okayama samples, the first letter stands for June $(\mathrm{J})$ and August (A); the second letter for silage (S), rumen fluid (R), and feces (F); and the number for a silage bag and dairy cow. The values in parentheses are the percentages of the variation accounted for by the component axes. 


\section{DISCUSSION}

Lactic acid was the predominant fermentation product in all the TMR silages analyzed, and the level appeared stable regardless of the product sampling season and the sampling region. Various homo-fermentative (P. acidilactici, P. ethanolidurans, L. acidipiscis, $L$. helveticus, L. plantarum, L. amylolyticus, L. acetotolerans, L. casei) and hetero-fermentative ( $L$. parafarraginis, L. buchneri, L. pontis, L. reuteri, L. suebicus, W. paramesenteroides) LAB were found in TMR silages; hence, composition of the LAB species was at least in part able to account for the acceptable lactic acid fermentation observed in the current study. Lactoba- cillus buchneri was found only in the Mie products, whereas $L$. pontis and $L$. reuteri were detected exclusively in the Okayama products. This difference in the hetero-fermentative LAB community did not explain the higher lactic-to-acetic acid ratio observed in the Mie products compared with the Okayama products. Silages containing L. buchneri should have a higher acetic acid level than those containing other heterofermentative LAB because L. buhcneri has a distinctive metabolism: it converts lactic acid into acetic acid and 1,2-propanediol (Oude Elferink et al., 2001). Except for the Mie November product, L. acetotolerans was found in all TMR silages regardless of the region of origin. Although we found L. acetotolerans frequently in bunker-

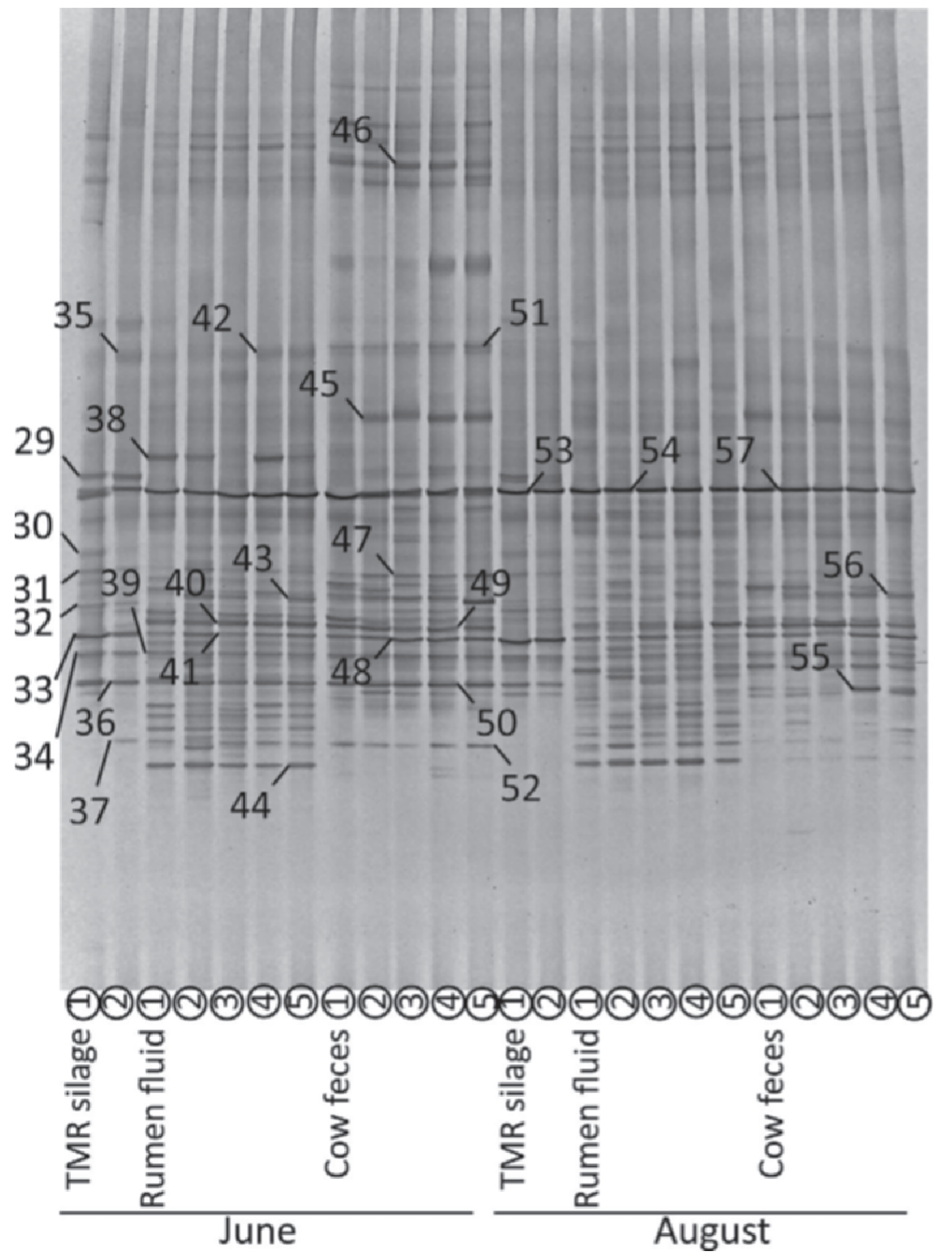

29 Lactobacillus pontis

30 Lactobacillus amylolyticus

31 Lactobacillus pontis

32 Lactobacillus pontis

33 Lactobacillus pontis

34 Lactobacillus reuteri

35 Lactobacillus plantarum

36 Weissella paramesenteroides

37 Lactobacillus acetotolerans

38 Uncultured bacterium

39 Uncultured bacterium

40 Lactobacillus ruminis

41 Uncultured bacterium

42 Lactobacillus plantarum

43 Uncultured bacterium

44 Lactobacillus ruminis

45 Lactobacillus acetotolerans

46 Uncultured bacterium

47 Lactobacillus fructivorans

48 Lactobacillus pontis

49 Lactobacillus ruminis

50 Lactobacillus reuteri

51 Uncultured bacterium

52 Lactobacillus ruminis

53 Lactobacillus acetotolerans

54 Lactobacillus acetotolerans

55 Uncultured bacterium

56 Lactobacillus pontis

57 Lactobacillus acetotolerans

Figure 3. Lactic acid bacteria communities of TMR silage, and rumen fluid and feces of dairy cows collected at 2 different times from the Okayama Prefecture Livestock Research Institute (Okayama, Japan). The seasons describe when the silage and feces were sampled; silage was produced 1 to 2 mo before the sampling time. 
made whole crop corn silage by DGGE analysis [Li and Nishino, 2011; Wang et al., 2014; H. Han, C. Wang (Okayama University, Okayama, Japan), Z. Yu (China Agricultural University, Beijing, China), Q. Xu (China Agricultural University), and N. Nishino, unpublished data], we did not find it in TMR silage (Wang and Nishino, 2010, 2013). Unlike the silages examined in our previous studies and also the Mie November product in the current study, the TMR silages harboring $L$. acetotolerans contained one or several crop silages as the ingredient. It is yet unclear, however, if crop silage ingredients may support the growth of L. acetotolerans in TMR silage.

In the Mie fecal samples, L. animalis, L. johnsonii, $L$. reuteri, and $L$. plantarum were found in all the dairy cows tested; thus, except for L. plantarum, the LAB species usually detectable in the gut were indeed seen in the present study (Krause et al., 2003; Hernandez et al., 2008; Nader-Macías et al., 2008). Lactobacillus acetotolerans, L. suebicus, and L. casei appeared to survive in the Mie dairy cow feces. Although these LAB species might be present as indigenous bacteria, their DNA was not detected in feces when dairy cows received TMR silage that did not contain those LAB species. Therefore, although the reason why differences in survival rates were seen remains unknown, certain silage LAB could survive the digestion process in the ruminant gut.

Following Okayama product DGGE gel analysis, which indicated the survival of LAB species (Figure 3), 8 bands commonly found in rumen fluid were cloned and identified, and 4 (bands 38, 39, 41, and 43) were classified as uncultured bacteria. Likewise, in our previous study, wherein the rumen fluid was sampled from goats receiving wilted Italian ryegrass silage, 4 of the 5 bands were identified as uncultured bacteria (Han et al., 2012). Because the proportion of identifiable bacteria in the rumen is as low as $20 \%$ of the total population (Cho et al., 2006; Lukás et al., 2010), our results for the low species identification were regarded as credible.

In the DGGE analysis, multiple bands detected at different migrating positions are sometimes identified as the same bacterial species; this was seen for $L$. acetotolerans, L. pontis, W. paramesenteroides, L. plantarum, L. reuteri, L. johnsonii, L. murinus, and L. ruminis in the current study. Multiple bands with different DNA sequences can be regarded as different strains inhabiting the same environment. In addition, multiple bands may also be seen for the same strain, as multiple heterogeneous $16 \mathrm{~S}$ rRNA gene copies may exist in a single bacterial strain. Furthermore, band duplication from the same DNA sequence occasionally appears in DGGE gels because heteroduplex molecules can form during the PCR amplification process (Muyzer and Smalla, 1998). Although these limitations might narrow the validity of DGGE in this evaluation, the finding that the DNA of silage LAB was not detected in feces when dairy cows did not receive the LAB species indicated the potential for survival in the gut.

If a high survival rate is a prerequisite to confer probiosis, the LAB species found in both silage and feces may be good candidates, as most of the DGGE bands from feces were seen also in the rumen fluid. Lactobacillus acetotolerans, L. pontis, L. casei, L. suebicus, and L. plantarum are worth examining further in this respect. In the meantime, the criteria for the screening of probiotic LAB for ruminants have yet to be defined. Differences were observed between the bacterial communities in the rumen fluid, the solid phase of the rumen, and rumen wall, whereas few LAB were found in the 3 fractions (Cho et al., 2006; Lukás et al., 2010). Mucosa-associated bacterial communities in the rumen, duodenum, and colon were also found to differ (Collado and Sanz, 2007); hence, further studies are required to determine the LAB associated with gut content and mucosa in various gut segments of silage-fed dairy cows.

This study demonstrated that, even when dairy cows receive silage as the sole diet, it would be hard for silage $\mathrm{LAB}$ to survive the digestion process in the gut. The gut LAB community seemed robust and not easily affected by that in the silage. Principal component analysis of the Okayama products indicated the presence of intercow variations depending on the sampling time. Lodge-Ivey et al. (2009) also found week-to-week fluctuations in the bacterial DGGE profiles of the rumen content; hence, the fate of the silage LAB in the gut might vary to some extent, even in the same cow. However, the finding that the LAB communities in the silage, rumen fluid, and feces were clearly separated indicated that it was difficult for the silage LAB to survive in the cow rumen or lower gut.

Of the $14 \mathrm{LAB}$ species found in TMR silage, 5 were detected in dairy cow feces, including 3 LAB species (L. acetotolerans, L. pontis, and L. casei) that had been detected in a previous study as well [H. Han, C. Wang (Okayama University, Okayama, Japan), Z. Yu (China Agricultural University, Beijing, China), Q. Xu (China Agricultural University), and N. Nishino, unpublished data]. About one-third of the silage LAB were found to have the potential to convey their probiotic function; hence, silage can be regarded as a good vehicle for the propagation and delivery of probiotic LAB. Moreover, among the $5 \mathrm{LAB}$ species assumed to survive in the gut, 3 (L. acetotolerans, L. pontis, and L. suebicus) have never been reported to inhabit silage by plateculture technique. Therefore, the finding that about one-third of the silage LAB can survive in the gut may 
stem from the use of culture-independent analysis in the current study. Recently, we isolated L. acetotolerans and L. pontis as slow-growing LAB from silage and feces of silage-fed dairy cows. Using these isolates and other nonsurviving species, we will examine if these LAB species indeed show high survival rates in the cow gut and if the survival varies depending on the species and strains.

\section{REFERENCES}

Cho, S. J., K. M. Cho, E. C. Shin, W. J. Lim, S. Y. Hong, B. R. Choi, J. M. Kang, S. M. Lee, Y. H. Kim, H. Kim, and H. D. Yun. 2006. $16 \mathrm{~S}$ rDNA analysis of bacterial diversity in three fractions of cow rumen. J. Microbiol. Biotechnol. 16:92-101.

Collado, M. C., and Y. Sanz. 2007. Quantification of mucosa-adhered microbiota of lambs and calves by the use of culture methods and fluorescent in situ hybridization coupled with flow cytometry techniques. Vet. Microbiol. 121:299-306.

Han, H., S. Takase, and N. Nishino. 2012. Survival of silage lactic acid bacteria in the goat gastrointestinal tract as determined by denaturing gradient gel electrophoresis. Lett. Appl. Microbiol. $55: 384-389$.

Hernandez, J. D., P. T. Scott, R. W. Shephard, and R. A. M. Al Jassim. 2008. The characterization of lactic acid producing bacteria from the rumen of dairy cattle grazing on improved pasture supplemented with wheat and barley grain. J. Appl. Microbiol. 104:1754-1763.

Krause, D. O., W. J. Smith, L. L. Conlan, J. M. Gough, M. A. Williamson, and C. S. Mc Sweeney. 2003. Diet influences the ecology of lactic acid bacteria and Escherichia coli along the digestive tract of cattle: Neural networks and 16S rDNA. Microbiology 149:57-65.

Li, Y., and N. Nishino. 2011. Monitoring the bacterial community of maize silage stored in a bunker silo inoculated with Enterococcus faecium, Lactobacillus plantarum and Lactobacillus buchneri. J. Appl. Microbiol. 110:1561-1570.

Lodge-Ivey, S. L., J. Browne-Silva, and M. B. Horvath. 2009. Technical note: Bacterial diversity and fermentation end products in rumen fluid samples collected via oral lavage or rumen cannula. J. Anim. Sci. 87:2333-2337.

Lukás, F., J. Šimůnek, J. Mrázek, and J. Kopečný. 2010. PCR-DGGE analysis of bacterial population attached to the bovine rumen wall. Folia Microbiol. (Praha) 55:345-348.
McAllister, T. A., K. A. Beauchemin, A. Y. Alazzeh, J. Baah, R. M. Teather, and K. Stanford. 2011. The use of direct fed microbials to mitigate pathogens and enhance production in cattle. Can. J. Anim. Sci. 91:193-211.

McDonald, P., A. R. Henderson, and S. J. E. Heron. 1991. The Biochemistry of Silage. Chalcombe Publications, Welton, Lincoln, UK

Muyzer, G., and K. Smalla. 1998. Application of denaturing gradient gel electrophoresis (DGGE) and temperature gradient gel electrophoresis (TGGE) in microbial ecology. Antonie van Leeuwenhoek 73:127-141.

Nader-Macías, M. E. F., M. C. Otero, M. C. Espeche, and N. C. Maldonado. 2008. Advances in the design of probiotic products for the prevention of major diseases in dairy cattle. J. Ind. Microbiol Biotechnol. 35:1387-1395.

Oude Elferink, S. J. W. H., J. Krooneman, J. C. Gottschal, S. F. Spoelstra, F. Faber, and F. Driehuis. 2001. Anaerobic conversion of lactic acid to acetic acid and 1,2-propanediol by Lactobacillus buchneri. Appl. Environ. Microbiol. 67:125-132.

Rodriguez-Palacios, A., H. R. Staempfli, T. Duffield, and J. S. Weese. 2009. Isolation of bovine intestinal Lactobacillus plantarum and Pediococcus acidilactici with inhibitory activity against Escherichia coli $\mathrm{O} 157$ and F5. J. Appl. Microbiol. 106:393-401.

Shito, H., N. Yamana, Y. Shibuya, and K. Takahashi. 2006. Development of the roll baler for chopped materials. Jpn. Agric. Res. Q. 40:233-237.

Wang, C., H. Han, X. Y. Gu, Z. Yu, and N. Nishino. 2014. A survey of fermentation products and bacterial communities in corn silage produced in a bunker silo in China. Anim. Sci. J. 85:32-36. http://dx.doi.org/10.1111/asj.12076.

Wang, C., and N. Nishino. 2010. Presence of sourdough lactic acid bacteria in commercial total mixed ration silage revealed by denaturing gradient gel electrophoresis analysis. Lett. Appl. Microbiol. 51:436-442.

Wang, C., and N. Nishino. 2013. Effects of storage temperature and ensiling period on fermentation products, aerobic stability and microbial communities of total mixed ration silage. J. Appl. Microbiol. 114:1687-1695.

Weinberg, Z. G., and R. E. Muck. 1996. New trends and opportunities in the development and use of inoculants for silage. FEMS Microbiol. Rev. 19:53-68.

Weinberg, Z. G., R. E. Muck, P. J. Weimer, Y. Chen, and M. Gamburg. 2004. Lactic acid bacteria used in inoculants for silage as probiotics for ruminants. Appl. Biochem. Biotechnol. 118:1-9. 